D. E. Blair

Nagoya Math. J.

Vol. 55 (1974) 1-3

\title{
ON THE ZEROS OF A CONFORMAL VECTOR FIELD
}

\author{
DAVID E. BLAIR(*)
}

\section{Introduction}

In [1] S. Kobayashi showed that the connected components of the set of zeros of a Killing vector field on a Riemannian manifold $\left(M^{n}, g\right)$ are totally geodesic submanifolds of $\left(M^{n}, g\right)$ of even codimension including the case of isolated singular points. The purpose of this short note is to give a simple proof of the corresponding result for conformal vector fields on compact Riemannian manifolds. In particular we prove the following

THEOREM. Let $\left(M^{n}, g\right)$ be a compact Riemannian manifold of dimension $n \geq 2$. Let $F$ be the set of zeros of a conformal vector field $\xi$ and let $F=\bigcup V_{i}$ where the $V_{i}$ 's are the connected components of $F$. Then each $V_{i}$ is either an umbilical submanifold of $\left(M^{n}, g\right)$ of even codimension including the case of isolated singular points or an isolated singular point of a conformal non-Killing vector field on a Euclidean sphere.

The idea of our proof is to reduce the problem to Kobayashi's case by a simple application of a theorem of M. Obata characterizing a sphere to conformality. In Section 2 we discuss Obata's result and then prove our theorem in Section 3.

\section{Preliminaries}

A Riemannian metric $\bar{g}$ is said to be conformal to $g$ if there exists a smooth function $\rho$ on $M^{n}$ such that $\bar{g}=e^{2 \rho} g$. Let $f: M^{n} \rightarrow M^{n}$ be a diffeomorphism of $M^{n}$ onto itself; we say $f$ is a conformal diffeomorphism if $f^{*} g$ is conformal to $g$.

Let $C\left(M^{n}, g\right)$ denote the Lie group of all conformal diffeomorphisms of $\left(M^{n}, g\right)$ and $C_{0}\left(M^{n}, g\right)$ the connected component of the identity. A

Received July 19, 1973.

(*) Partially supported by NSF Grant GP-36684. 
subgroup $G$ of $C\left(M^{n}, g\right)$ is said to be essential if it does not become a group of isometries under any conformal change of metric, and a conformal vector field is said to be essential if its one-parameter group is essential. In [2] and [3] M. Obata obtained the following results.

THEOREM (Obata [2]). Let $\left(M^{n}, g\right)$ be a compact Riemannian manifold of dimension $n>2$. Then $C_{0}\left(M^{n}, g\right)$ is essential if and only if $\left(M^{n}, g\right)$ is conformally diffeomorphic to a Euclidean sphere.

THEOREM (Obata [3]). Let $\xi$ be an essential conformal vector field on a Euclidean sphere. Then $\xi$ has either exactly one or exactly two singular points.

\section{Proof of the Theorem}

The proof of the theorem is, for $n>2$, by cases using Obata's result. If $C_{0}\left(M^{n}, g\right)$ is inessential, then there exists a conformal change of metric, say $\bar{g}=e^{2 \rho} g$, such that $C_{0}\left(M^{n}, g\right)$ is a group of isometries with respect to $\bar{g}$. Thus given a conformal vector field $\xi$ on $\left(M^{n}, g\right), \xi$ is Killing on $\left(M^{n}, \bar{g}\right)$ and hence by Kobayashi's Theorem each $V_{i}$ is a totally geodesic submanifold of $\left(M^{n}, \bar{g}\right)$ of even codimension. Thus it remains only to show that $V_{i}$ is umbilical in $\left(M^{n}, g\right)$. To this end let $\nabla$ and $\bar{\nabla}$ be the Riemannian connexions of $g$ and $\bar{g}$ respectively and let $P=\operatorname{grad} \rho$. Then

$$
\bar{\nabla}_{X} Y=\nabla_{X} Y+(X \rho) Y+(Y \rho) X-g(X, Y) P .
$$

Now let $`$ denote the imbedding of $V_{i}$ in $M^{n}$. Considering $V_{i}$ as a submanifold of $\left(M^{n}, g\right)$ with $g^{\prime}$ and $\nabla^{\prime}$ denoting the induced Riemannian metric and connexion, choose a local orthonormal frame $\eta_{1}, \cdots, \eta_{k}$ of normal vector fields on $V_{i}, k=\operatorname{codim} V_{i}$, and let $h^{\alpha}$ denote the corresponding second fundamental forms. Then the Gauss equation is

$$
\nabla_{\iota * X^{\iota}{ }_{*}} Y=\iota_{*} \nabla_{X}^{\prime} Y+h^{\alpha}(X, Y) \eta_{\alpha}
$$

summed on $\alpha$. Considering $V_{i}$ as a submanifold of $\left(M^{n}, \bar{g}\right)$ with $\bar{V}^{\prime}$ denoting the induced Riemannian connexion, the Gauss equation is

$$
\bar{\nabla} \iota_{* X} \iota_{*} Y=\iota_{*} \bar{\nabla}_{X}^{\prime} Y
$$

Thus using (3.1), (3.2) and (3.3) we have

$$
\iota_{*} \nabla_{X}^{\prime} Y+h^{\alpha}(X, Y) \eta_{\alpha}=\iota_{*} \bar{\nabla}_{X}^{\prime} Y-\left(\iota_{*} X \rho\right) \iota_{*} Y-\left(\iota_{*} Y \rho\right) \iota_{*} X+g\left(\iota_{*} X, \iota_{*} Y\right) P .
$$


Now taking the $g$ inner product with $\eta_{\beta}$ we have

$$
h^{\beta}(X, Y)=\left(\eta_{\beta} \rho\right) g^{\prime}(X, Y)
$$

and hence that $V_{i}$ is umbilical in $\left(M^{n}, g\right)$.

If on the other hand $C_{0}\left(M^{n}, g\right)$ is essential then $\left(M^{n}, g\right)$ is conformally diffeomorphic to a Euclidean sphere, but a conformal vector field remains conformal under a conformal change of metric. Thus if a conformal vector field $\xi$ is essential, its zeros are isolated. If $\xi$ is inessential then again there exists a conformal change of metric with respect to which $\xi$ becomes a Killing vector field.

Finally if $n=2$, there exist local isothermal parameters with respect to which $\left(M^{2}, g\right)$ becomes a Hermitian (complex) manifold. If now $\xi$ is a conformal vector field on $\left(M^{2}, g\right)$, the conformal transformations of its oneparameter group are given by analytic functions. Thus by the identity theorem for analytic functions, their fixed points are isolated and hence the zeros of $\xi$ are isolated

\section{REFERENCES}

[1] S. Kobayashi, Fixed points of isometries, Nagoya Math. J. 13 (1958) 63-68.

[2] M. Obata, The conjectures on conformal transformations of Riemannian manifolds, J. Diff. Geom. 6 (1971) 247-258.

[ 3 ] M. Obata, Conformal transformations of Riemannian manifolds, J. Diff. Geom. 4 (1970) 311-333.

Michigan State University 